\title{
Caracterización citogenética de Hoplosternum littorale (Pisces, Siluriformes) del río Riachuelo (Corrientes, Argentina)
}

\author{
Caramello, C.S.; Sánchez, S.; Jorge, L.C. \\ Instituto de Ictiología, Facultad de Ciencias Veterinarias, Universidad Nacional del Nordeste, Sargento Cabral \\ 2139, Corrientes (3400), Argentina. Tel/fax 03783-425753. E-mail: cynsolcar@hotmail.com.
}

\begin{abstract}
Resumen
Caramello, C.S.; Sánchez, S.; Jorge, L.C.: Caracterización citogenética de Hoplosternum littorale (Pisces, Siluriformes) del río Riachuelo (Corrientes, Argentina). Rev. vet. 24: 1, 29-31, 2013. La especie Hoplosternum littorale es conocida vulgarmente con el nombre de "cascarudo". Se caracteriza por tener una amplia distribución en América del Sur. Tres ejemplares de la población del río Riachuelo (dos hembras y un macho) fueron citogenéticamente analizados y se caracterizaron por presentar un número diploide de 60 cromosomas. La formúla cariotípica estuvo constituida por 8 cromosomas meta-submetacéntricos y 52 acrocéntricos, con un número fundamental de 68 en ambos sexos. No se observaron diferencias cromosómicas entre sexos. La impregnación con nitrato de plata identificó la ocurrencia de NOR simples, localizadas en el brazo corto del par número 5. El bandeo $\mathrm{C}$ reveló una gran cantidad de bloques de heterocromatina presentes en la mayoría de los cromosomas, preferentemente situados en regiones terminales e intersticiales adyacentes al centrómero. Los datos obtenidos corroboran que $H$. littorale posee una evolución cariotípica conservada, siendo necesario complementar los estudios citogenéticos con marcadores moleculares para una mejor comprensión de las relaciones evolutivas y el mantenimiento de la macroestructura cariotípica de la especie.
\end{abstract}

Palabras clave: Hoplosternum littorale, cariotipo, bandeo-C, NOR, Argentina.

\begin{abstract}
Caramello, C.S.; Sánchez, S.; Jorge, L.C.: Cytogenetic analysis of Hoplosternum littorale (Pisces, Siluriformes) from Riachuelo river (Corrientes, Argentina). Rev. vet. 24: 1, 29-31, 2013. Hoplosternum littorale, commonly known as "armored catfishes", are widely distributed in South America. Three specimens captured in Riachuelo river (two females and a male) were cytogenetically analyzed. Fishes were characterized to have 60 chromosomes. Karyotype formula was made up with 8 meta-submetacentrics and 52 acrocentric chromosomes, the fundamental number was 68 in both sexes, and there were no chromosomic differences between them. Just one pair of subtelo-acrocentrics chromosomes showed NOR with silver nitrate impregnation, situated on the short arm of pair five. C-banding reveled a great amount or chromatin blocks in most of the chromosomes; they were located in subterminal and interstitial regions. These data reveal that $H$. littorale has a conserved karyotypic evolution. The cytogenetic studies should be complemented by molecular markers for further understanding of the evolutionary relationships and the maintenance of the karyotypic macroestructure of the species.
\end{abstract}

Key words: Hoplosternum littorale, karyotype, C-banding, NOR, Argentina.

\section{INTRODUCCIÓN}

La familia Callichthyidae es considerada monofilética según estudios basados en caracteres morfológicos y moleculares, comprendiendo dos grupos naturales Corydoradinae (Aspidoras, Scleromystax y Corydoras) y Callichthyinae (Callichthys, Dianema, Hoplosternum,

Recibido: 20 septiembre 2012 / Aceptado: 8 noviembre 2012
Lepthoplosternum y Megalechis) ${ }^{1,16,20}$. La especie Hoplosternum littorale es conocida vulgarmente con el nombre de "cascarudo". Se caracteriza por tener una amplia distribución en América del Sur, localizándose en las cuencas de los ríos Paraguay, Paraná, Uruguay, Delta del río Paraná y Esteros del Iberá ${ }^{2}$.

Los ejemplares de $H$. littorale son de mediano tamaño y se caracterizan morfológicamente por presentar dos hileras de placas óseas en el flanco. Los huesos 
coracoides se observan mucho más expuestos en la región ventral. La aleta dorsal presenta una espina fuerte y ocho radios. La aleta caudal es profundamente cóncava, presenta una coloración pardo oliva homogénea. Se alimenta de cladóceros, copépodos, dípteros quironómidos y nemátodos, así como de detritus orgánicos ${ }^{17}$.

Desde el punto de vista citogenético la familia Callichthyidae se distingue por presentar una gran variabilidad, tanto numérica como morfológica; el número diploide varía de $2 \mathrm{n}=40$ en Corydoras nattereri a $2 \mathrm{n}=132$ en Corydoras aeneus ${ }^{12}$. Además se observa la ocurrencia de polimorfismos cromosómicos numéricos y estructurales ${ }^{7}$.

Debido a los escasos datos citogenéticos existentes para $H$. littorale, el objetivo del presente trabajo fue realizar la caracterización de la estructura cariotípica de la especie, a partir del análisis de ejemplares del río Riachuelo, afluente del río Paraná en la localidad de Corrientes, Argentina.

\section{MATERIAL Y MÉTODOS}

Se analizaron tres ejemplares de $H$. littorale (dos hembras y un macho). Tras su captura, los peces fueron transportados en bolsas de polietileno que contenían agua y oxígeno. Durante el traslado se tomaron todos los recaudos para que los animales lleguen en óptimas condiciones al laboratorio. Antes del sacrificio los peces fueron anestesiados con metasulfonato de tricaína MS222 (Finquel $囚)$.

Para el estudio de los cromosomas se utilizó la porción anterior del riñón ${ }^{10}$. Los cromosomas mitóticos se obtuvieron a través de la disociación del órgano en cubetas que contenían $10 \mathrm{ml}$ de solución de Hank, posteriormente se continuó con los procedimientos de rutina ${ }^{5}$. Las regiones organizadoras de nucleolos (NOR) se identificaron por tinción con nitrato de plata (AgNOR) ${ }^{6}$. La heterocromatina constitutiva se localizó por el método convencional de bandeo $\mathrm{C}^{22}$.

Las metafases fueron fotografiadas con una cámara digital Infinity 1 acoplada a un microscopio óptico Olympus BX41, y posteriormente se analizaron con el programa analizador de imágenes Image ProPlus 5.1. En el cariotipo se distribuyeron los pares homólogos en orden decreciente de tamaño. Los cromosomas se clasificaron como metacéntricos/submetacéntricos (M-SM), y subtelocéntricos/acro- céntricos (ST-A) de acuerdo a su morfología y relación de brazos ${ }^{9}$.

\section{RESULTADOS Y DISCUSIÓN}

La evaluación citogenética realizada a $H$. littorale permitió determinar un número diploide de 60 cromosomas. La fórmula cariotípica estuvo constituida por 8 $\mathrm{M} / \mathrm{SM}+52 \mathrm{~A}$, con un número fundamental (FN) de 68 en ambos sexos (Figura 1A). No se observaron diferencias cromosómicas entre sexos. Este número diploide es coincidente con el obtenido en otras poblaciones originarias de distintas cuencas hidrográficas de Brasil y Venezuela. Sin embargo, algunos informes muestran leves variaciones en torno a la fórmula cariotípica, probablemente debido a diferencias en la condensación de los cromosomas $3,8,11,13-15,18,19,21$.

La impregnación con nitrato de plata identificó la NOR simple, localizada en el brazo corto del par número 5 , siendo coincidente con la constricción secundaria. (Figura 1A recuadro). Nuestros resultados concuerdan con lo expuesto por otros autores que describen una NOR simple en posición terminal en cromosomas ST/A $3,8,11,13,18,19,21$. Por otro lado, se evidenció un hete-

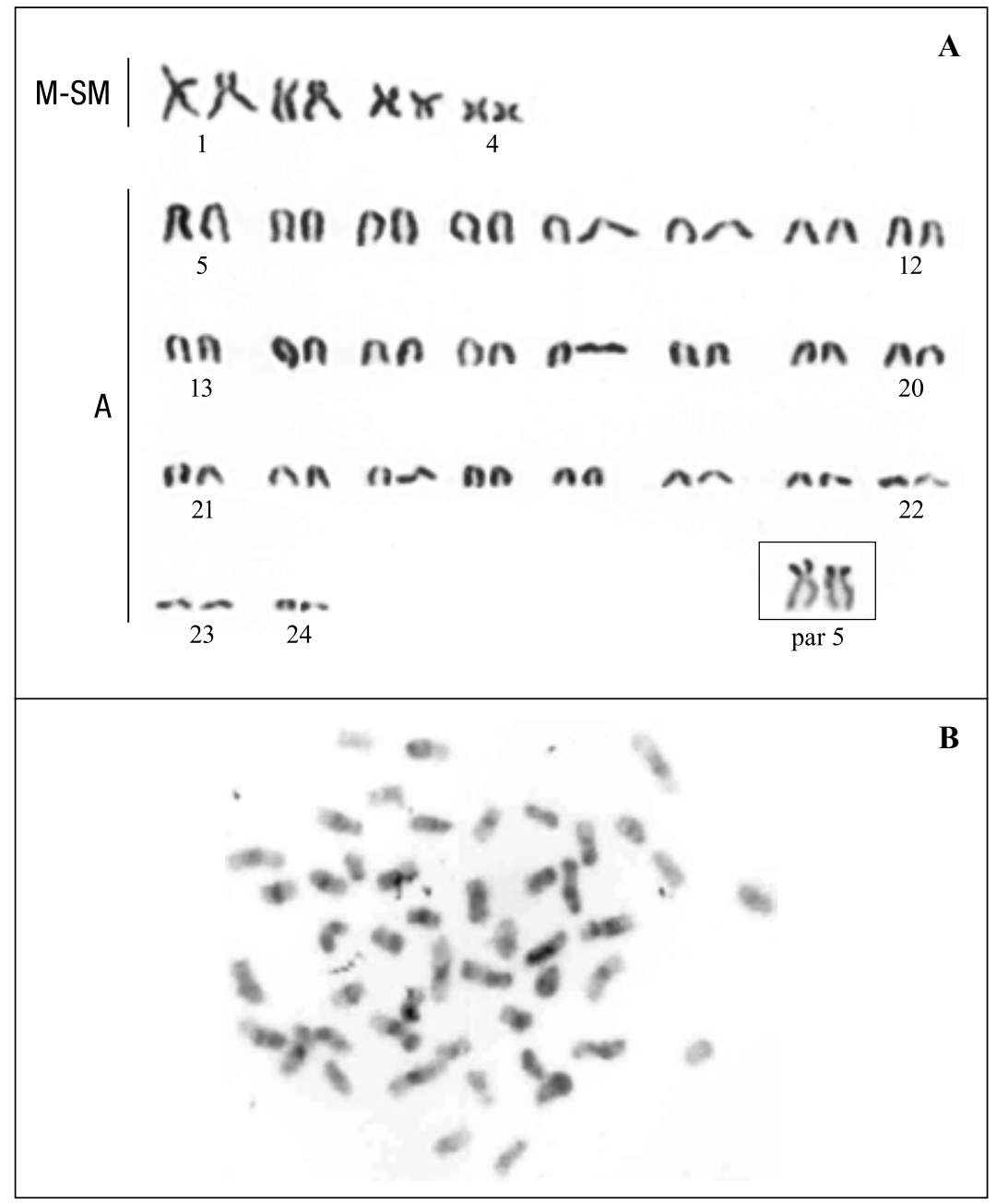

Figura 1. (A): cariotipo de $H$. littorale con coloración convencional de Giemsa; en el recuadro se destacan los cromosomas portadores de NOR (par 5). (B): bandeo C. 
romorfismo en el tamaño de las regiones NOR+ con la presencia de una marcación mayor en uno de los cromosomas y otra pequeña en su homólogo. Este fenómeno posiblemente se relacione con un número diferente de copias de genes ribosómicos en los homólogos resultantes de entrecruzamiento desigual o algún otro tipo de reordenamiento cromosómico ${ }^{4}$.

El bandeo $\mathrm{C}$ reveló una gran cantidad de bloques de heterocromatina presentes en la mayoría de los cromosomas, preferentemente situados en regiones terminales e intersticiales adyacentes al centrómero (Figura 1B). Similares resultados se encontraron en las poblaciones de $H$. littorale analizadas hasta el momento ${ }^{3,8}$, 11, 13, 19,21.

En conclusión, los datos obtenidos refuerzan la idea de que $H$. littorale posee una evolución cariotípica conservada repecto al número diploide, estructura cariotípica, número y posición de NOR y patrón de distribución de los segmentos heterocromáticos, siendo necesario complementar los estudios citogenéticos con análisis moleculares para una mejor comprensión de las relaciones evolutivas y el mantenimiento de la macroestructura cariotípica de la especie.

\section{REFERENCIAS}

1. Britto MR. 2003. Phylogeny of the subfamily Corydoradinae Hoedeman, 1952 (Siluriformes: Callichthyidae), with a definition of its genera. Proceed Acad Nat Sci Philadelphia 153: 119-154.

2. Casciota J, Almirón A, Bechara J. 2003. Los peces de la laguna Iberá, Ed. Colección Universitaria, Buenos Aires, $131 \mathrm{p}$.

3. Coelho PN, Farías LN, Nagamachi CY, Barros RM, Pieczarka JC. 2000. Análise citogenética na espécie Hoplosternum littorale (Pisces, Siluriformes, Callichthyidae) proveniente do rio Peixe-Boi-PA. VIII Simp Citogenet de Peixes, Manaus, Brasil, p.79.

4. Foresti F, Almeida LF, Toledo AS. 1981. Polymorphic nature of nucleolus organizer regions in fishes. Cytogen \& Cell Gen 31: 137-144.

5. Foresti F, Oliveira C, Almeida LF. 1993. A method for chromosome preparations from large specimens of fishes using in vitro short treatment with colchicine. Experientia 49: 810-813.

6. Howell, WM, Black DA. 1980. Controlled silver-staining of nucleolus organizer regions with a protective colloidal developer: A 1-step method. Experientia 6: 1014-1015.

7. Kavalco KF, Margarido VP. 2001. Polimorfismo cromossomico em Callichthys callichthys (Pisces, Siluriformes, Callichthyidae) da bacia do rio Paraná. $47^{\circ}$ Congresso Nacional de Genética, Águas de Lindóia, SP, Brasil, 279.

8. Konerat JT. 2008. Relações filogenéticas em Callichthyinae (Siluriformes Callichthyidae). Uma contribuição citogenética aos estudos evolutivos. Tesis de Mestrado, Universidad Estadual de Maringá, PR, Brasil, 29 p.
9. Levan A, Fredga K, Sandberg AA. 1964. Nomenclature for centromeric position on chromosomes. Hereditas 52: 201-220.

10. Moreira-Filho O, Bertollo LA. 1991. Extraction and use of the cephalic kidney for chromosome studies in small fish. Brazil J Genet 14: 1085-1090.

11. Nirchio M, Pérez J, Granado A, Ron E. 2006. Conventional karyotype, constitutive heterochromatin, and nucleolar organizer regions in Hoplosternum littorale (Pisces: Callichthyidae) from Caicara del Orinoco, Venezuela. Agrobiología 18: 113-116.

12. Oliveira C, Almeida LF, Foresti F, Britski HA, Toledo AS. 1988 Chromosome formulae of neotropical freshwater fishes. Genet Mol Biol 11: 577-624.

13. Pazza R, Kavalco KF, Almeida LF, Bertollo LC. 2005. Hoplosternum littorale (Teleostei, Callichthyidae) from a Coastal River basin in Brazil. Cytogenetic analysis and gene mapping of 5S and 18S rDNA. Caryologia 58: 339344.

14. Portela AL, Natali MR, Júlio HF. 1990. Estudos meióticos na espécie Hoplosternum littorale (Callichthyidae, Siluriformes). III Simpósio de Citogenética Evolutiva de Peixes Neotropicais, Botucatu, SP, Brasil, p. 28.

15. Porto JI, Feldberg E. 1992. Comparative cytogenetic study of the armored catfishes of the genus Hoplosternum (Siluriformes, Callichthyidae). Braz J Genet 15: 359-367.

16. Reis RE. 1998. Anatomy and phylogenetic analysis of the neotropical callichthyid catfishes (Ostariophysi, Siluriformes). Zool J Linnean Soc 124: 105-168.

17. Ringuelet RA, Arámburu RH, Arámburu AA. 1967. Los peces argentinos de agua dulce, Public. Comisión de Investigaciones Científicas y Técnicas, Buenos Aires, 602 p.

18. Rosa R, Kavalco KF, Margarido VP. 2002. Estudos citogenéticos comparativos em duas populações de $\mathrm{Ho}$ plosternum littorale (Pisces, Siluriformes, Callichthyidae) coletadas no rio Paraná (Guaíra/PR) e no rio Corumbá (Corumbá/MS). IX Simpósio de Citogenética de Peixes, Maringa, PA, Brasil, p. 36.

19. Santi AP, Martins IC. 2002. Análise citogenética en Hoplosternum littorale (Pisces, Siluriformes, Callichthyidae) de ocorrência no ribeirão Maringá, Paraná. IX Simpósio de Citogenética de Peixes, Maringa, PA, Brasil, p. 83.

20. Shimabukuro CK, Oliveira C, Reis RE, Foresti F. 2004. Molecular phylogeny of the armored catfish family Callichthyidae (Ostariophysi, Siluriformes). Molec Phylogen \& Evol 32: 152-163.

21. Shimabukuro CK, Oliveira C, Foresti F. 2005. Comparative cytogenetic studies in species of the subfamily Callichthyinae (Teleostei: Siluriformes: Callichthyidae). Caryologia 58: 102-111.

22. Sumner AT. 1972. A simple technique for demonstrating centromeric heterochromatin. Exp Cell Res 75: 304-306. 